VITAL

VOICES

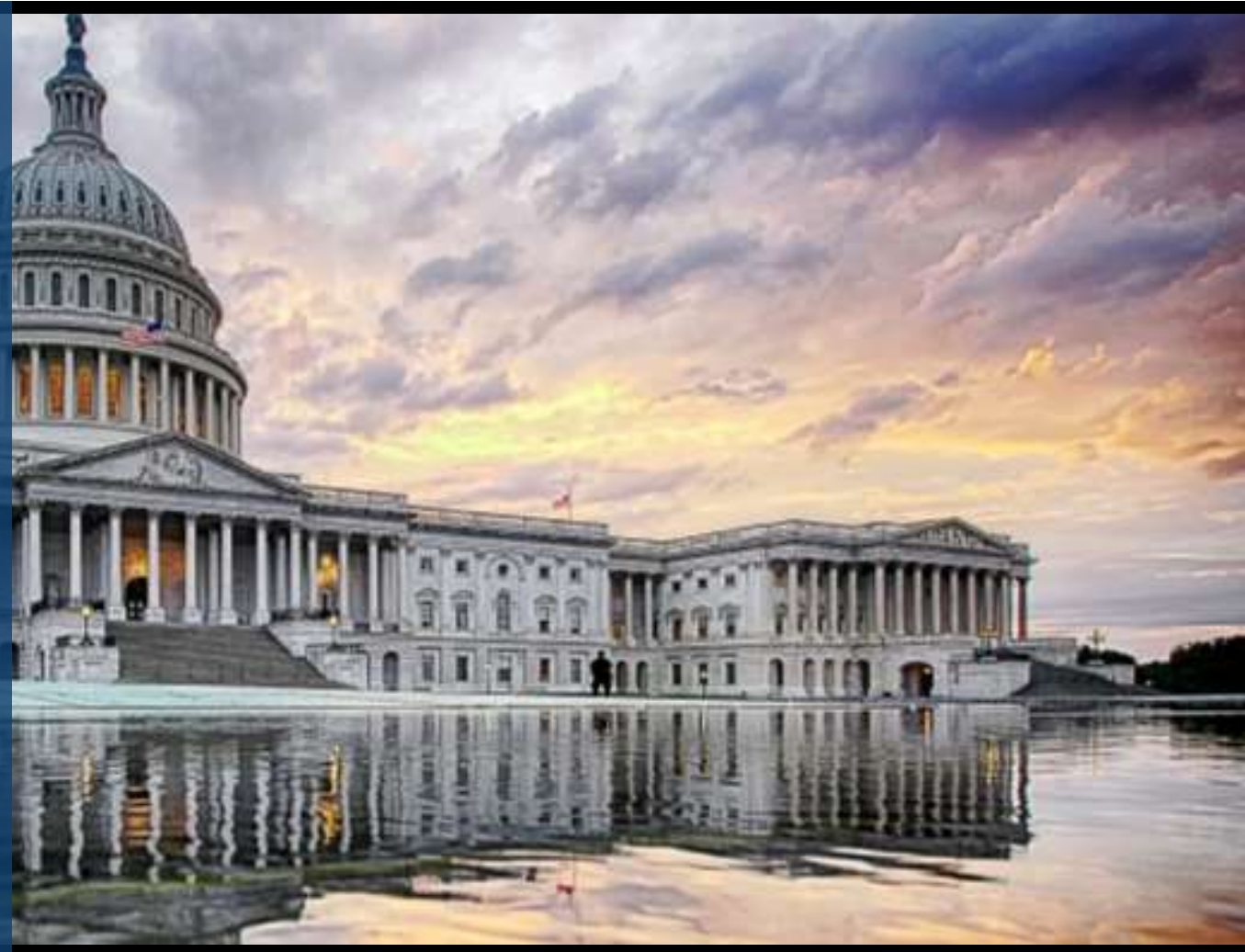

Issues of Importance to District of Columbia Adults Age 45+

\section{Health Care}

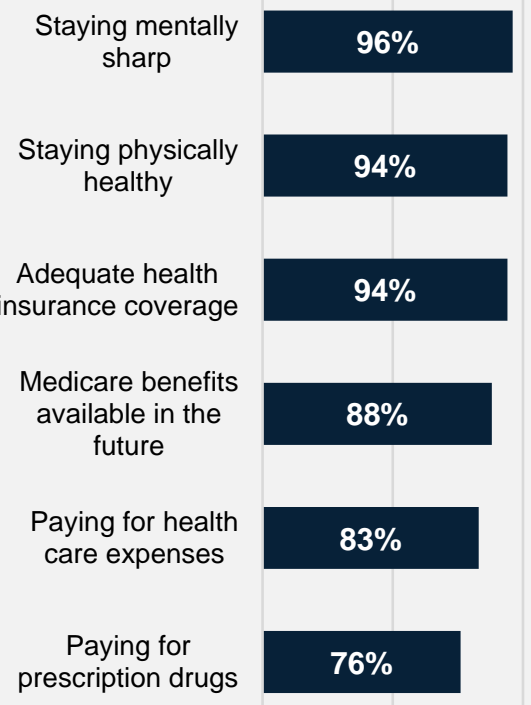

$0 \% \quad 50 \% \quad 100 \%$

\section{Economic/Financial}

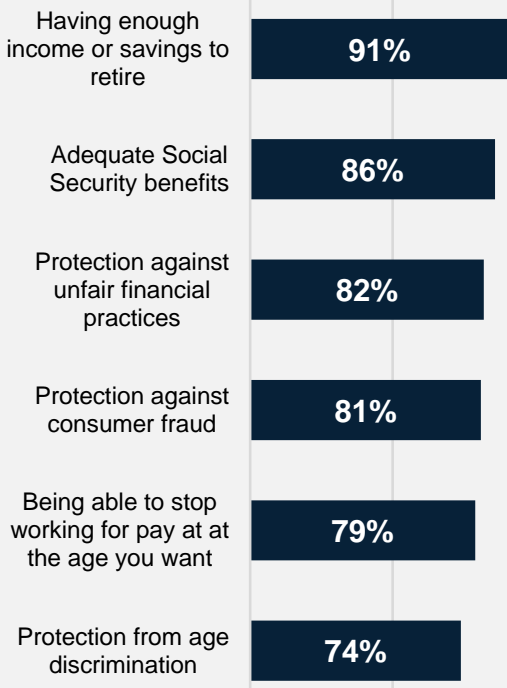

\section{Independent Living/ Long-Term Care}

Having high quality long-term care $84 \%$

Getting to the places you need to go independently

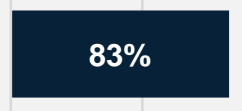

Caring for a loved one

$81 \%$
Staying in your own home as you get older

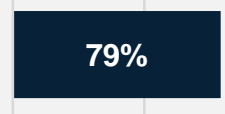

$0 \%$

$50 \% \quad 100 \%$ 
Vital Voices: Issues That Impact District of Columbia Adults Age 45 and Older, October 2021

Interviews were conducted with 704 residents of the District of Columbia by landline (55\%), cell phone (33\%), and online (13\%) between September 30, 2021 and October 12, 2021.

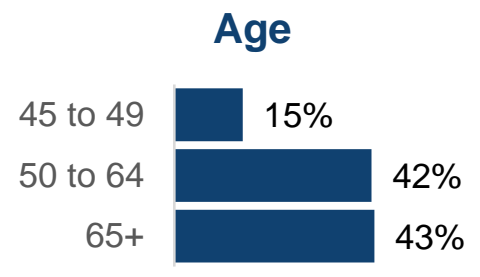

Employment

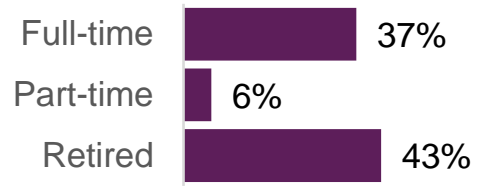

\section{Gender}

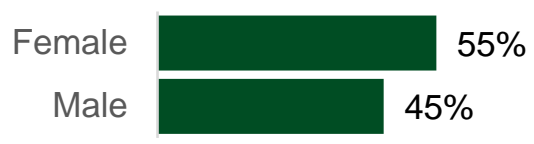

\section{Race/Ethnicity}

Black/ African American

White/ Caucasian

Other

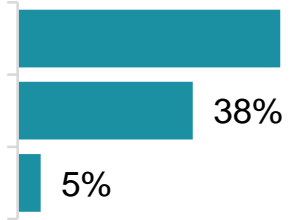

Political Views

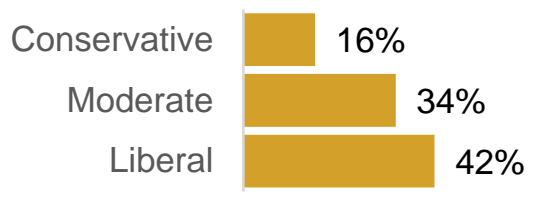

\section{Education}

Some college or less

4-year college degree

Post-graduate study or degree

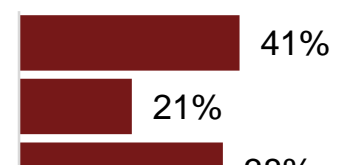

$38 \%$

\section{Marital Status}

Married/ living with partner

Divorced/ separated

Widowed

Never married

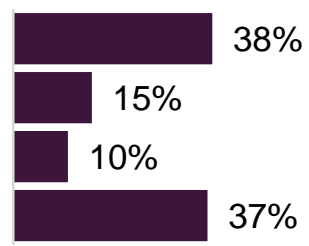

$37 \%$

\section{Annual Household Income}

Less than $\$ 50,000$

$\$ 50,000$ to less than $\$ 100,000$

$\$ 100,000+$

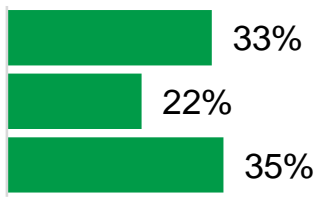

$57 \%$

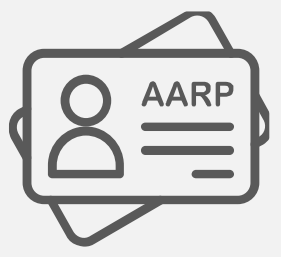

$43 \%$

AARP Member

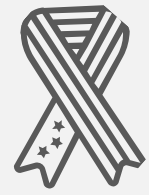

$10 \%$

Veteran 